\title{
Sports-Related Injuries of the Pediatric Musculoskeleton
}

\author{
Lower Extremity
}

\author{
Kirsten Ecklund
}

\section{Learning Objectives}

- To recognize unique injuries of the pediatric musculoskeleton commonly associated with athletic activity.

- To differentiate these pathologic entities from imaging features associated with normal growth and development.

\subsection{Epiphyseal and Osteochondral Injuries}

\subsubsection{Osteochondritis Dissecans}

Osteochondritis dissecans (OCD) was first described in 1888 by Franz Konig [1] as a cause of loose bodies within the joint. Historically, juvenile and adult-onset OCD were felt to represent the same process but with a better prognosis in skeletally immature patients. While the pathogenesis and natural history of juvenile OCD (JOCD) remain uncertain, most agree that it is acquired likely due to repetitive trauma and that it is distinct from osteochondral lesions seen in adults. The term JOCD should be reserved for patients with open physes, typically between the ages of 10 and 15 years. Surgical versus conservative management remains controversial due to the ambiguity around etiology and prognosis. Juvenile OCD of the lower extremity is most frequently encountered in the femoral condyles and the talar dome. Several reports including those from the multi-institutional Research in Osteochondritis dissecans of the Knee (ROCK) Group [2] suggest that the lesion originates within the unos-

K. Ecklund $(\bowtie)$

Department of Radiology, Boston Children's Hospital, Boston, MA, USA

e-mail: kirsten.ecklund@ childrens.harvard.edu sified epiphyseal cartilage with subsequent involvement of the subchondral bone and potentially the articular cartilage [3]. Radiographic findings may include subchondral lucency, marginal sclerosis, and osteochondral bony sequestrum partially or fully separated from the parent bone (Fig. 19.1a).

Numerous MR imaging classifications, most notably the De Smet classification [4], exist with the aim of assessing stability of the OCD. These have primarily focused on breach of the articular cartilage and fluid insinuating between the parent bone and the bony progeny as signs of instability. More recently, high-resolution MR with detailed description of the lesion is being used to assess healing and prognosis. The chondral "fragment" is better termed the progeny of the parent bone, and ossification of the progeny is a sign of healing. Fluid signal intensity sandwiched between the parent bone and unossified epiphyseal cartilage, linear vertical low signal intensity in the overlying articular cartilage, and full-thickness defects are poor prognostic signs (Fig. 19.1b).

Normal developmental ossification of the femoral condyles may be confused with OCD, though it is typically seen in children. Radiographically the femoral condylar ossification centers may appear irregular, fragmented, and spiculated. MR imaging features of developmental ossification rather than OCD include location in the posterior condyle as opposed to the intercondylar notch, accessory ossification centers, a large amount of residual epiphyseal cartilage, and lack of bone marrow edema [5] (Fig. 19.2). Additionally, disruption of the trilaminar secondary ossification center (SOC) physis overlying chondral abnormality, best seen on fatsuppressed fluid-sensitive sequences, is indicative of JOCD [6] (Figs. 19.1 and 19.2). This finding is most helpful in young children with substantial residual epiphyseal cartilage.

In children less than 6 years of age, physiologic T2 hyperintensity will be seen in the posterior epiphyseal cartilage of both medial and lateral femoral condyles, likely related to advancing ossification within the cartilaginous epiphysis. 


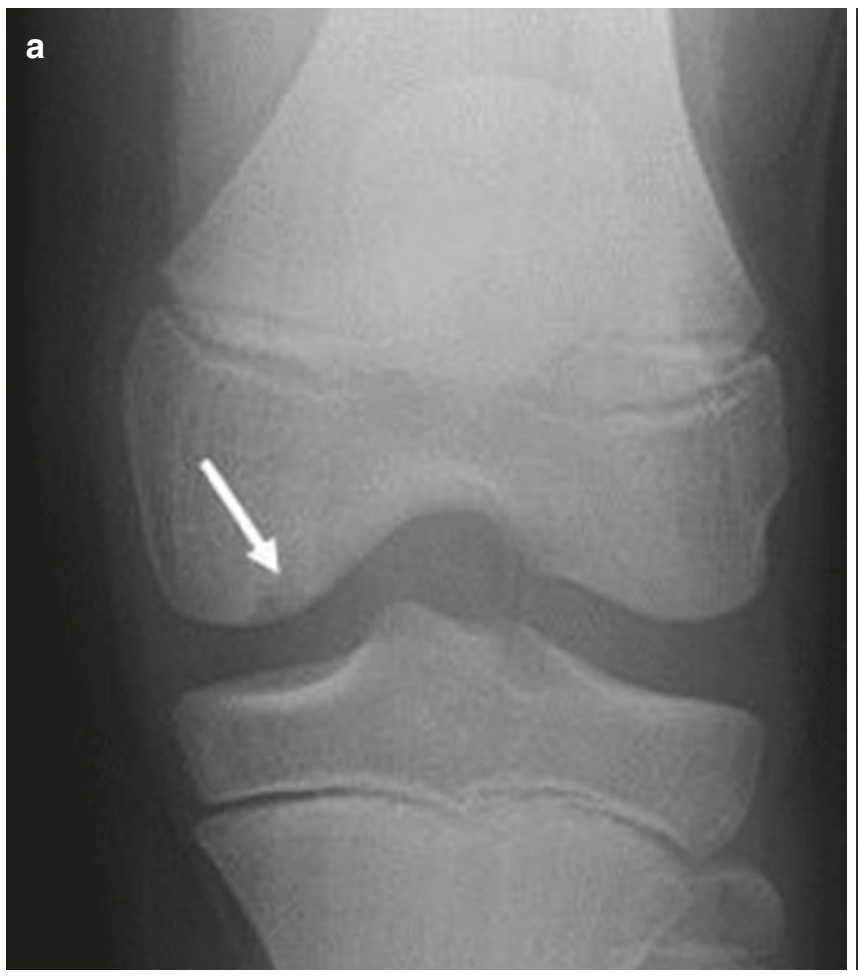

Fig. 19.1 (a, b) Osteochondritis dissecans in a 10-year-old male soccer player with knee pain. The frontal radiograph (a) shows subchondral lucent lesion with marginal sclerosis involving the weight-bearing, intercondylar notch region of the medial femoral condyle (white arrow). The sagittal T2-weighted image with fat suppression (b) shows

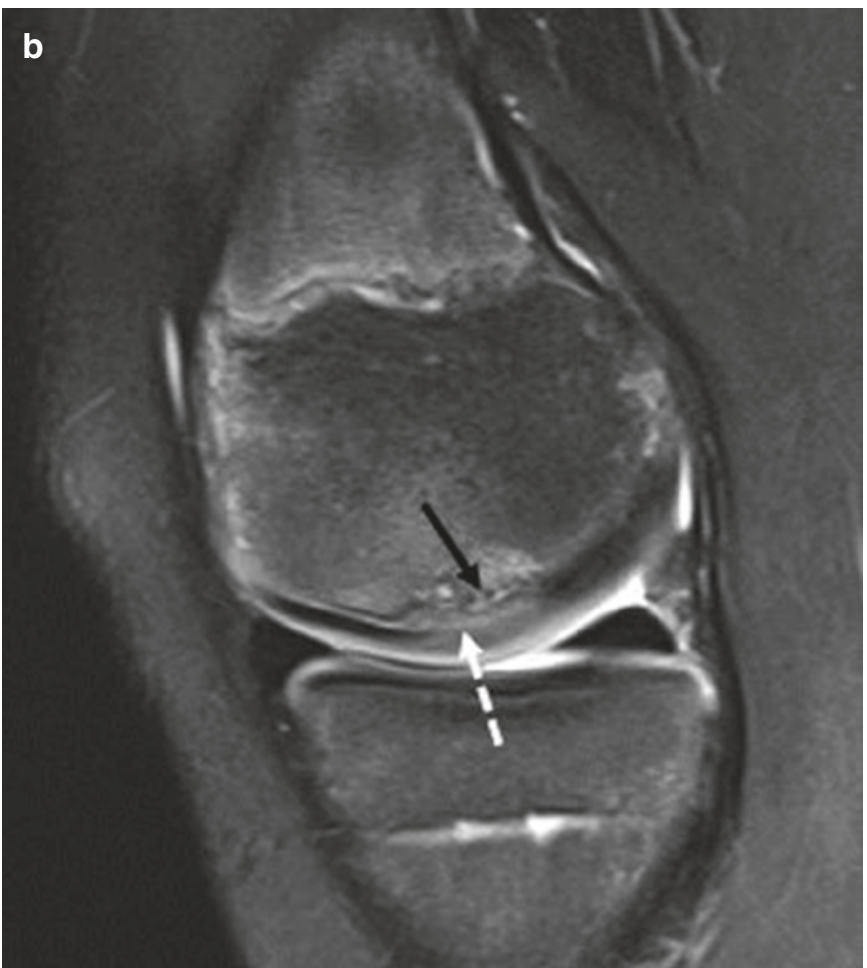

increased signal in the overlying epiphyseal cartilage, disruption of the overlying secondary physis (white dashed arrow), and subchondral marrow edema. Hyperintense T2 signal at the bone-cartilage interface (black arrow) and subchondral fibrovascular foci are further signs of JOCD and likely indicate poorer prognosis
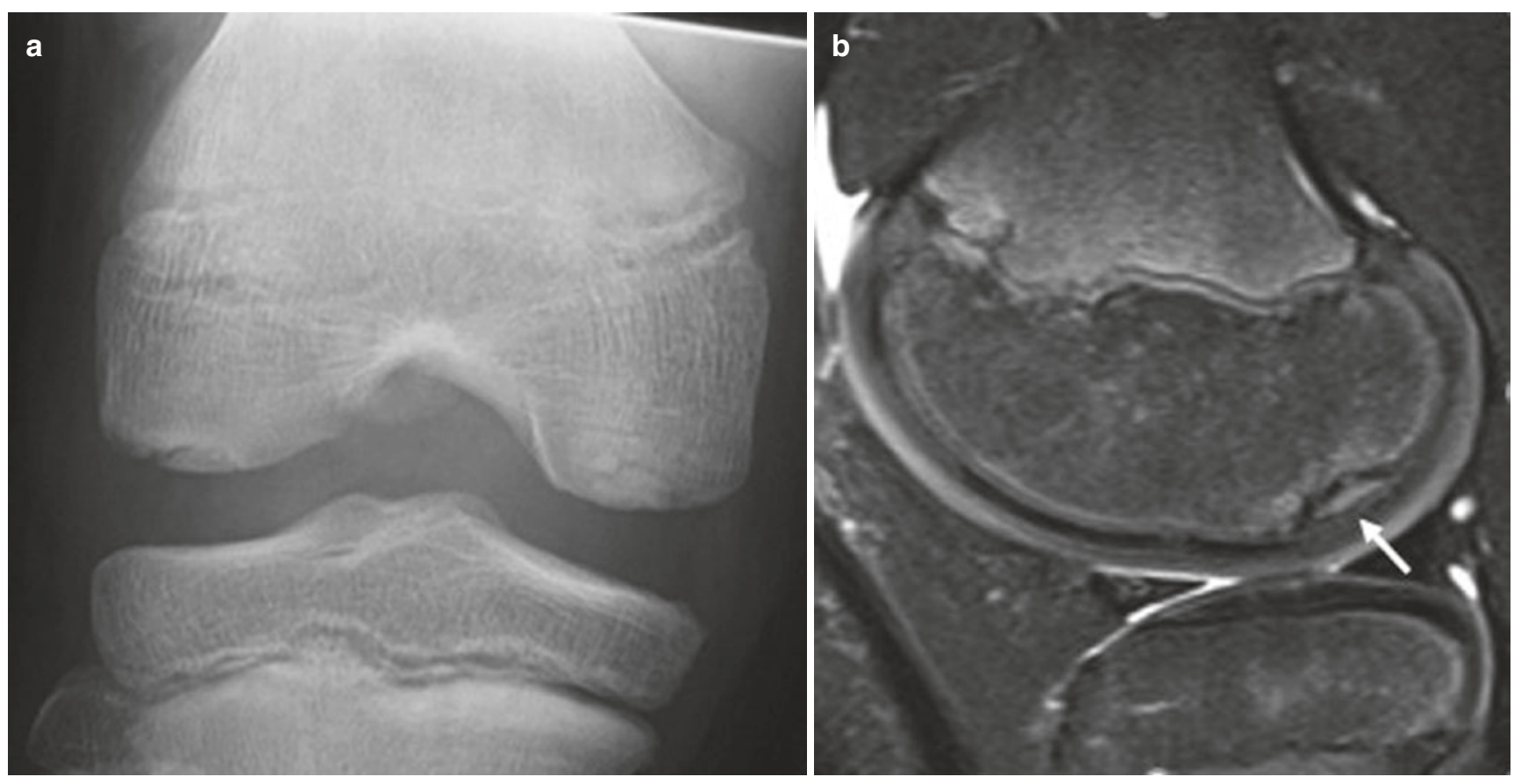

Fig. 19.2 (a, b) Normal developmental ossification in a 12-year-old baseball player with anterior knee pain after sliding into base. The frontal radiograph (a) and sagittal T2-weighted image with fat suppression (b) show osteochondral irregularity in the non-weight-bearing, poste- rior aspects of the medial and lateral femoral condyles. The MR features which favor developmental ossification rather than JOCD include posterior location, intact overlying secondary physis (arrow), and minimal subchondral marrow edema 


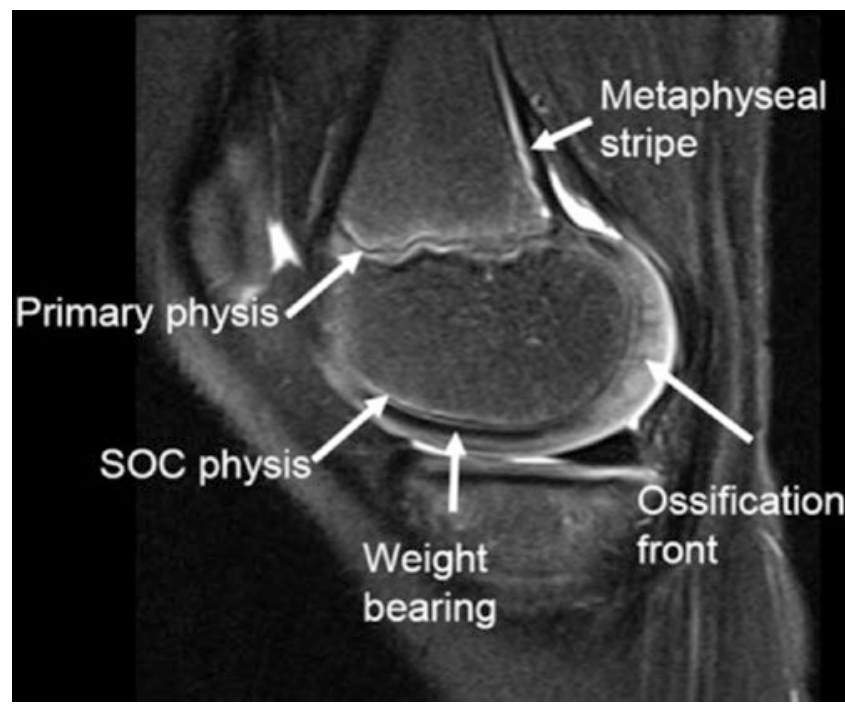

Fig. 19.3 Normal MR signal variation in a 7-year-old boy. Sagittal T2-weighted image with fat suppression shows the normal trilaminar primary and secondary (SOC) physes. Low signal intensity in the weight-bearing epiphyseal cartilage is thought to be due to desiccation of free water, while higher signal in the posterior cartilage relates to the metabolically active ossification front. The image also shows the normal hyperintense metaphyseal stripe reflecting vascular tissue between the periosteum and subchondral bone. Axially, this structure is circumferential and has the appearance of a metaphyseal ring or collar (not shown)

In these young children, low signal intensity is normally seen in the inferior aspect of the cartilaginous epiphysis, presumably due to weight-bearing [7]. This normal maturation process should not be confused with osteochondral injury (Fig. 19.3). Additionally, physiologic focal increased fluid signal intensity in the epiphyseal cartilage is common just prior to ossification and has been termed the "pre-ossification center" [8]. Misinterpretation of developmental variants as OCD may, in part, account for the higher rate of spontaneous resolution of juvenile OCD compared to the adult form.

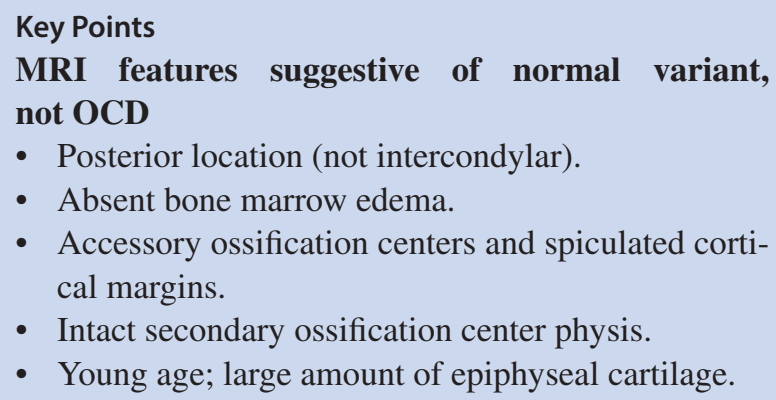

- Accessory ossification centers and spiculated cortical margins.

- Intact secondary ossification center physis.

- Young age; large amount of epiphyseal cartilage.

\subsubsection{Acute Osteochondral Fractures}

Acute traumatic chondral injuries are significantly more common than meniscal and anterior cruciate ligament injuries in the immature knee. In patients with open physes, femoral condylar injuries are more common than patellar and femoral trochlea [9]. Low-grade chondral injuries can be subtle with chondral thickening and abnormal signal intensity. MR has a higher sensitivity for the detection of more severe lesions with full-thickness chondral or osteochondral fracture. Subchondral bone marrow edema, best seen on fatsuppressed fluid-sensitive sequences, should alert the radiologist to scrutinize the overlying articular cartilage for associated injury (Fig. 19.4). The images should be scrutinized for free intra-articular fragments. In contrast to OCD, these acute injuries always involve the articular cartilage with or without extension to the epiphyseal cartilage and subchondral bone. As a reminder, T2 hyperintensity in the femoral condylar epiphyseal cartilage posteriorly in young children represents normal signal variation associated with the advancing ossification front [10] and should not be misinterpreted as chondral injury.

\subsection{Avulsion Injuries}

Avulsion injuries in children are most frequent during puberty and adolescence, when the physis is the weakest region of the musculoskeleton. In this age group, acute apophyseal avulsion fractures are more common than ligament and tendon injury. Unlike epiphyses, apophyses are growth centers which do not contribute to linear growth and are sites of tendon or ligament attachment. Acute, displaced apophyseal avulsion fractures may result from sudden, forceful muscle contraction. In contrast, myotendinous forces applied to the apophysis due to repetitive activity may lead to microavulsion at the interface between the bony apophysis and adjacent cartilaginous physis. Associated inflammation identified on MR imaging as increased fluid signal in the apophysis, physis, and soft tissues is termed traction apophysitis [11]. Acute and repetitive overuse apophyseal injuries are most common in the pelvis but are also commonly seen at the knee and ankle.

Acute patellar sleeve fractures, named for the sleeve of unossified cartilage which surrounds the osseous patella, are avulsion injuries at the inferior and rarely superior poles of the patella at the sites of patellar and quadriceps tendon attachment which occur with forceful quadriceps contraction against resistance, usually with a flexed knee. Radiographic features of the much more common inferior patellar sleeve 

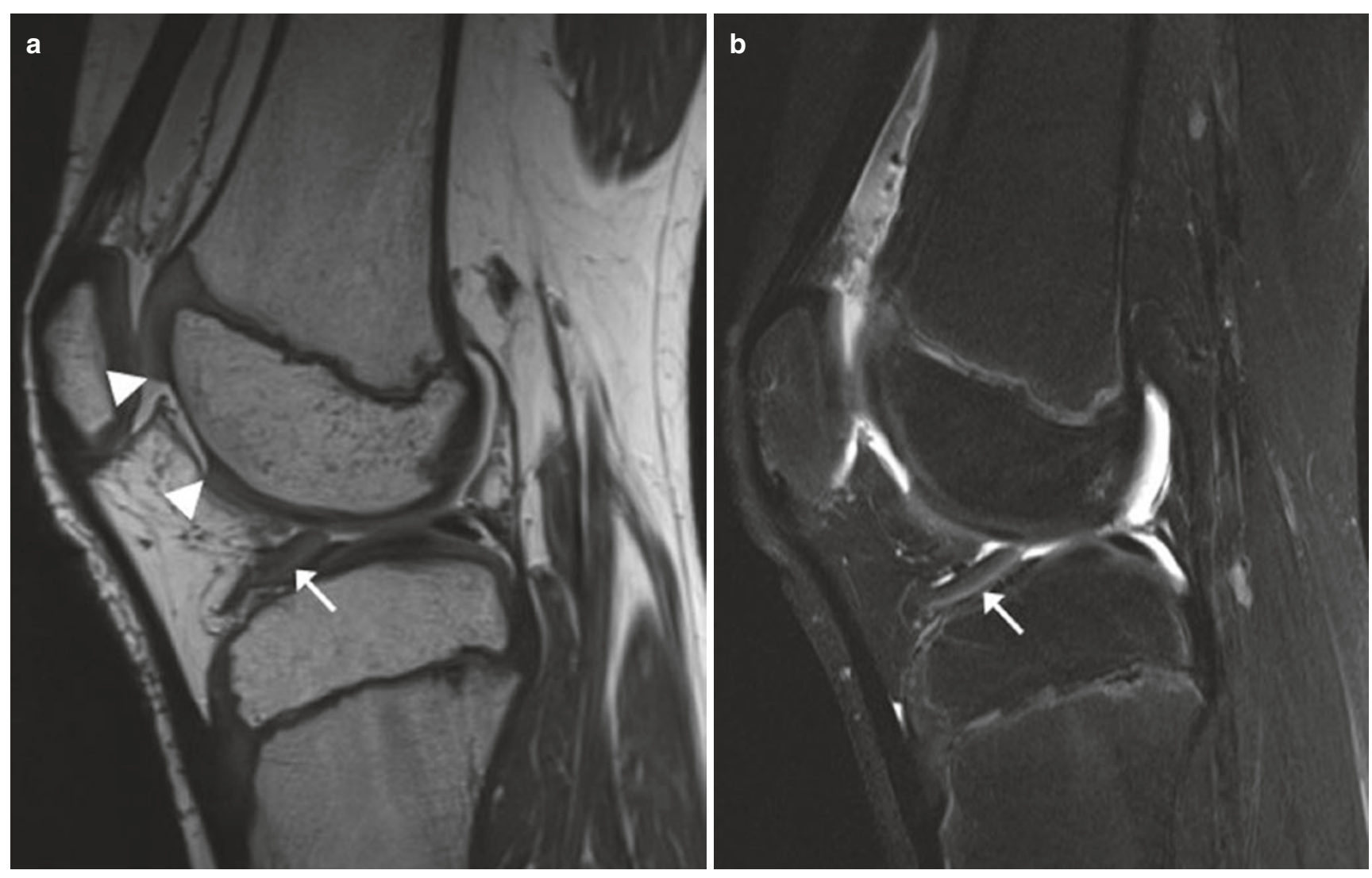

Fig. 19.4 (a, b) Acute osteochondral fracture in an 11-year-old boy who fell onto his knee playing soccer. Sagittal proton density WI (a) and T2-weighted image with fat suppression (b) reveal a large osteochondral defect (margins marked by arrowheads) in the anterior medial femoral condyle. The displaced osteochondral fracture fragment is in the anterior joint, sitting atop the tibial plateau (arrow). Note the absence of subchondral marrow edema related to normally limited epiphyseal vascularity fracture include patella alta, a sliver-like patellar fracture fragment, joint effusion, and soft tissue swelling (Fig. 19.5a, b). MRI maybe performed to confirm the integrity of the patellar tendon and will also show the chondral component of the fracture, which if large is an indicator of operative fixation. Chronic avulsive stress at the same location results in inferior patellar traction apophysitis, called SindingLarsen-Johansson disease. The MR and radiographic appearances can be similar to a nondisplaced patellar sleeve fracture; however, there is no history of an acute traumatic event (Fig. 19.5c, d).

Apophyseal injury at the opposite end of the patellar tendon, the tibial tubercle insertion, can also be acute leading to a Salter-Harris type II or III fracture through the tubercle physis or chronic resulting in Osgood-Schlatter traction apophysitis (Fig. 19.5e). Radiographs show soft tissue swelling overlying the tibial tubercle, while MR images show marrow edema in the tibial tubercle and fluid signal in the distal patellar tendon and infrapatellar bursa. Fragmentation of the tubercle often reflects normal developmental ossification and should not be used to make the diagnosis of OsgoodSchlatter. The term "jumper's knee," used to describe patellar tendon pathology, should be reserved for skeletally mature patients with fused physes.

Traction apophysitis at the Achilles tendon calcaneal insertion is termed Sever's disease. Calcaneal apophyseal sclerosis is a manifestation of physiologic stress associated with weight-bearing and is not an indication of apophysitis. As with Osgood-Schlatter, MR imaging features include fluid in the distal Achilles tendon and the adjacent retrocalcaneal bursa, overlying soft tissue swelling, and marrow edema within the apophysis.

While the incidence of anterior cruciate ligament (ACL) tears in adolescent athletes, especially females, has increased dramatically in the last two decades, acute avulsion fracture of the tibial eminence ACL attachment is common in younger skeletally immature children, especially boys ages $8-14$ years old, when the intercondylar eminence is incompletely ossified [12]. The Meyers and McKeever classification is most commonly used to describe the epiphyseal osteochondral displacement: type I, nondisplaced; type II, posteriorly hinged attachment with elevation of the anterior margin of the fragment; type III, completely displaced; and type IV, comminuted or rotated fragment [13]. MR images should be 


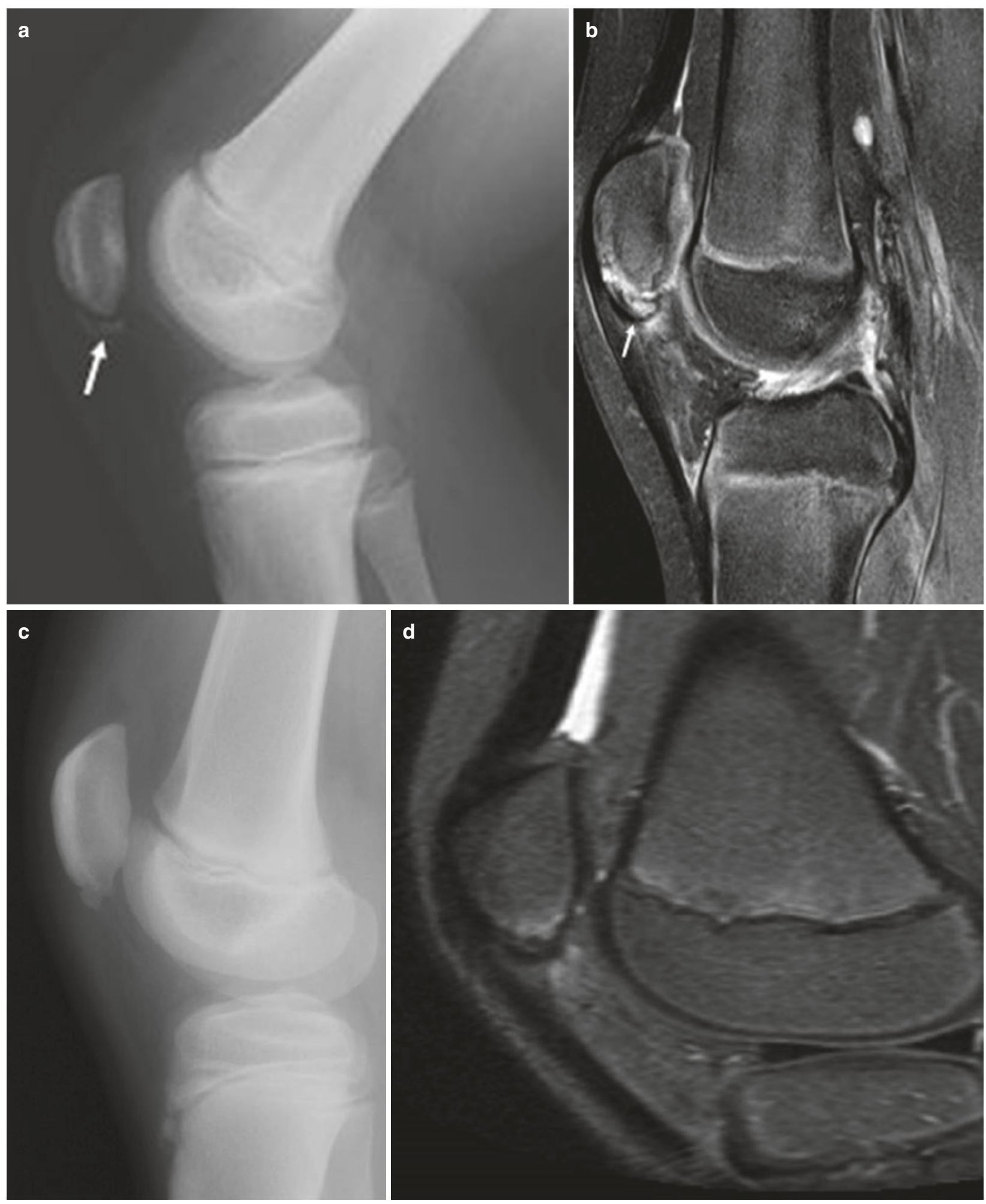

Fig. 19.5 (a, b) Acute patellar sleeve frature: Lateral radiograph (a) and sagittal T2-weighted image with fat suppression (b) in 11 y.o boy with acute anterior knee pain after landing on his flexed knee when tackled from behind in football show a thin inferior patellar fracture fragment (arrow) and associated marrow and soft tissue. The patellar tendon is intact. (c, d) Proximal patellar tendon traction apophysitis, Sinding-Larsen-Johansson: Lateral radiograph (c) and sagittal T2-weighted image with fat suppression (d) in a 9 y.o. female ice skater with months of inferior patellar pain show fragmentation and marrow edema in the inferior patella and as well as subtle edema in the proximal patellar tendon and in the superior aspect of Hoffa fat pad. The history differentiates this from patellar sleeve fracture. (e) Distal patellar tendon traction apopysitis, Osgood-Schlatter: The final sagittal T2-weighted image with fat suppression (E) in 13 y.o. hockey player with 6 weeks of tibial tubercle pain, tenderness to palpation, and recent growth spurt shows intense marrow edema within a hypertrophied tibial tubercle. Associated edema is present in the distal patellar tendon and inferior Hoffa fat pad 


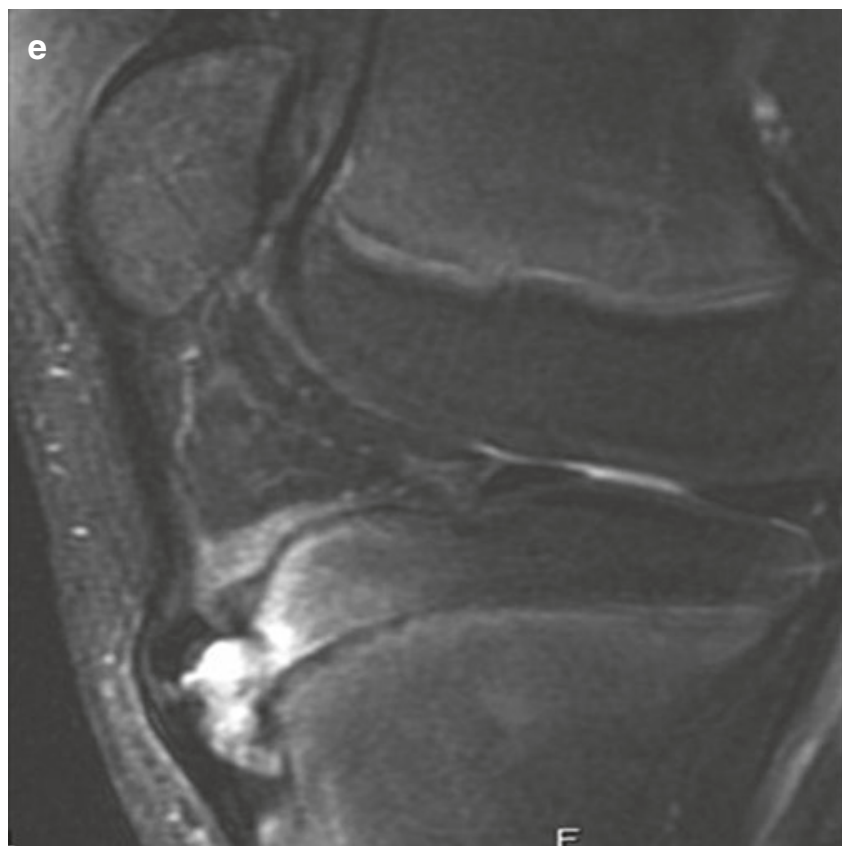

Fig. 19.5 (continued)

scrutinized for impingement of the transverse intermeniscal ligament, anterior horn of the medial meniscus, or anterior horn of the lateral meniscus between the fracture fragment and the donor site, impediments to closed reduction (Fig. 19.6).

\section{Key Points}

\section{Traction Apophysitis}

- Patients must have open physes.

- MR shows edema in the apophysis and overlying soft tissues and fluid in the tendon at the apophyseal attachment.

- Fragmentation or irregularity of the apophysis can be normal.

- Acute apophyseal fractures may have a similar appearance and are distinguished by history of trauma.

\subsection{Physeal Fractures}

The physis, or growth plate, is an exclusively cartilaginous structure throughout growth. As such, it is the weakest component of the bone-joint unit in children. Mechanical forces which result in ligamentous or tendinous injury in adults tend to cause physeal fractures in children. This is especially true in early adolescence when the physis becomes wider due to increased metabolic activity associated with the phys- iologic growth spurt. Simultaneous increased athletic endeavors in many middle and upper school children further predisposes to growth plate fractures [8]. The Salter-Harris classification of growth plate fractures was initially described in 1963 and remains widely used. Type I is a fracture confined to the physis. Type II involves the physis and metaphysis. Type III extends from the epiphysis into the epiphysis. Type IV is an obliquely oriented fracture which extends through both the metaphysis and epiphysis, traversing the physis. Type $\mathrm{V}$ is a crush injury of the physis [14]. More than $30 \%$ of pediatric long bone fractures involve the physis with approximately $15 \%$ going on to develop post-traumatic growth arrest [15]. The risk of growth disturbance is less related to the Salter-Harris type than to the specific physis with lower extremity locations and those with undulating physes most at risk. When the fracture plane separates the physis from the epiphysis and disrupts the blood supply, vascular communications between the epiphysis and metaphysis may develop allowing a bony bridge to form across the physis. Large, central bridges lead to limb shortening, while smaller, peripheral bridges lead to angular deformity. In the lower extremity, physeal fractures are most common in the distal tibia with up to a third resulting in growth arrest (Fig. 19.7)

Distal femoral and proximal tibial physeal fractures are often radiographically occult, identified only when MR imaging is performed for persistent post-traumatic knee pain. These images should be scrutinized for widening and increased fluid signal within the physis and disruption of the perichondrium indicating nondisplaced fracture. Associated stripped periosteum can become entrapped within the injured physis and be an impediment to closed reduction [16] (Fig. 19.8).

\subsection{Physeal Overuse Stress Injury}

Growth disturbance can also occur from chronic repetitive overuse trauma in young athletes. Physeal dysfunction without premature transphyseal bone bridge formation occurs secondary to recurrent microtrauma leading to vascular compromise and disruption of enchondral ossification. Unossified bands or tongues of hypertrophic physeal cartilage extend into the metaphysis. Radiographically, this appears as physeal widening and irregularity. MR images in these patients show focal or band-like physeal widening and $\mathrm{T} 2$ hyperintensity related to the excess unossified cartilage as well as edema. The normal trilaminar appearance of the physis is disrupted in the area of injury. The most well-described locations of these injuries are the distal radius and olecranon in gymnasts and the proximal humerus in pitchers; however, similar physeal stress injury occurs in the knees in child athletes involved in football, basketball, soccer, gymnastics, and tennis [17]. Similarly, MR images in these children with knee pain show focal bands of physeal cartilage signal 

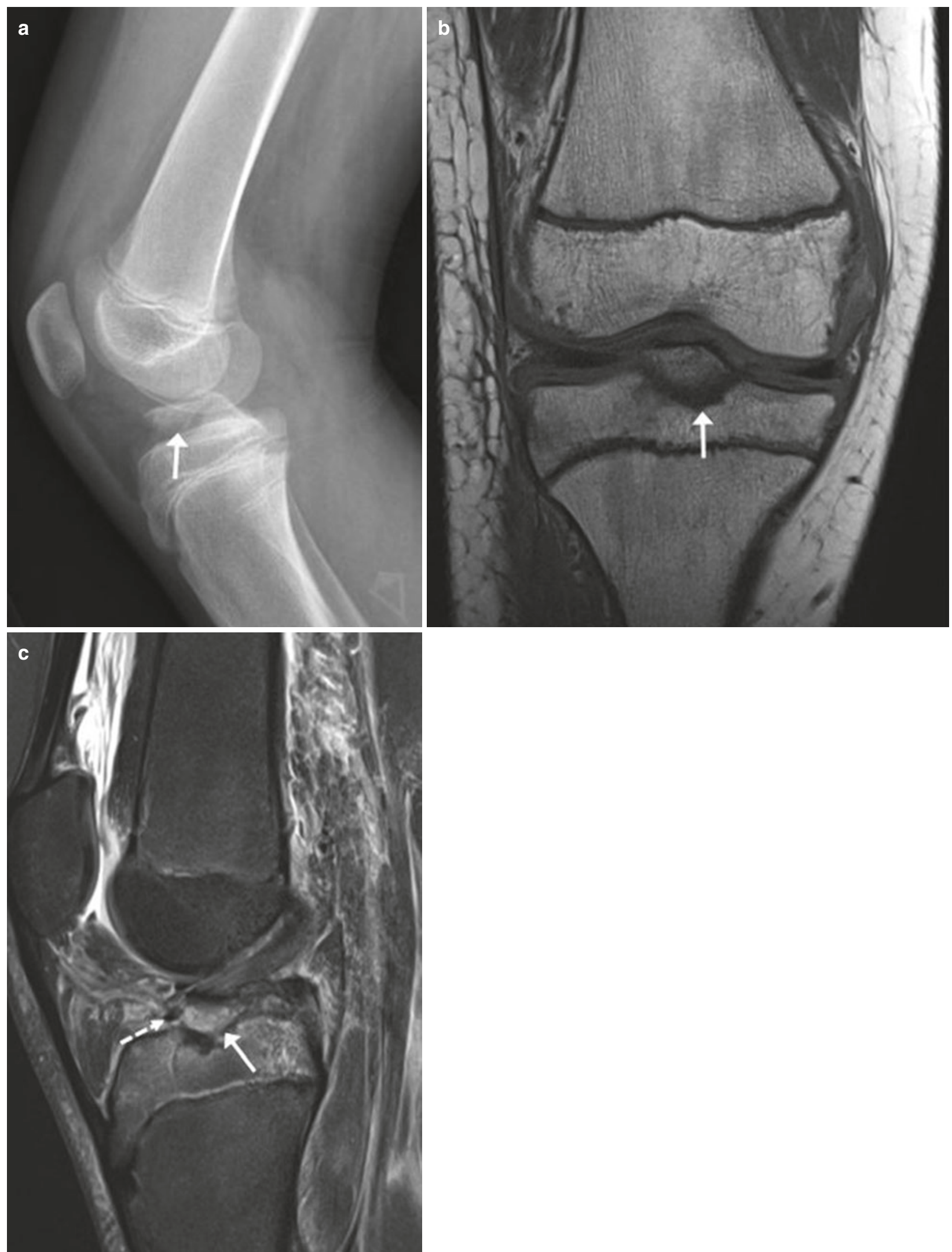

Fig. 19.6 (a-c) Acute tibial eminence avulsion fracture. Lateral radiograph (a), coronal T1-weighted image (b), and sagittal T2-weighted image with fat suppression (c) in a 12-year-old girl who crashed while ski racing. The tibial spine fracture plane is clearly delineated (arrows).
The anterior cruciate ligament is intact and attached to the avulsed uplifted, displaced fragment (Meyers-McKeever type III). Note the transverse meniscal ligament interposed between the fracture fragment and donor site anteriorly (dashed arrow) 


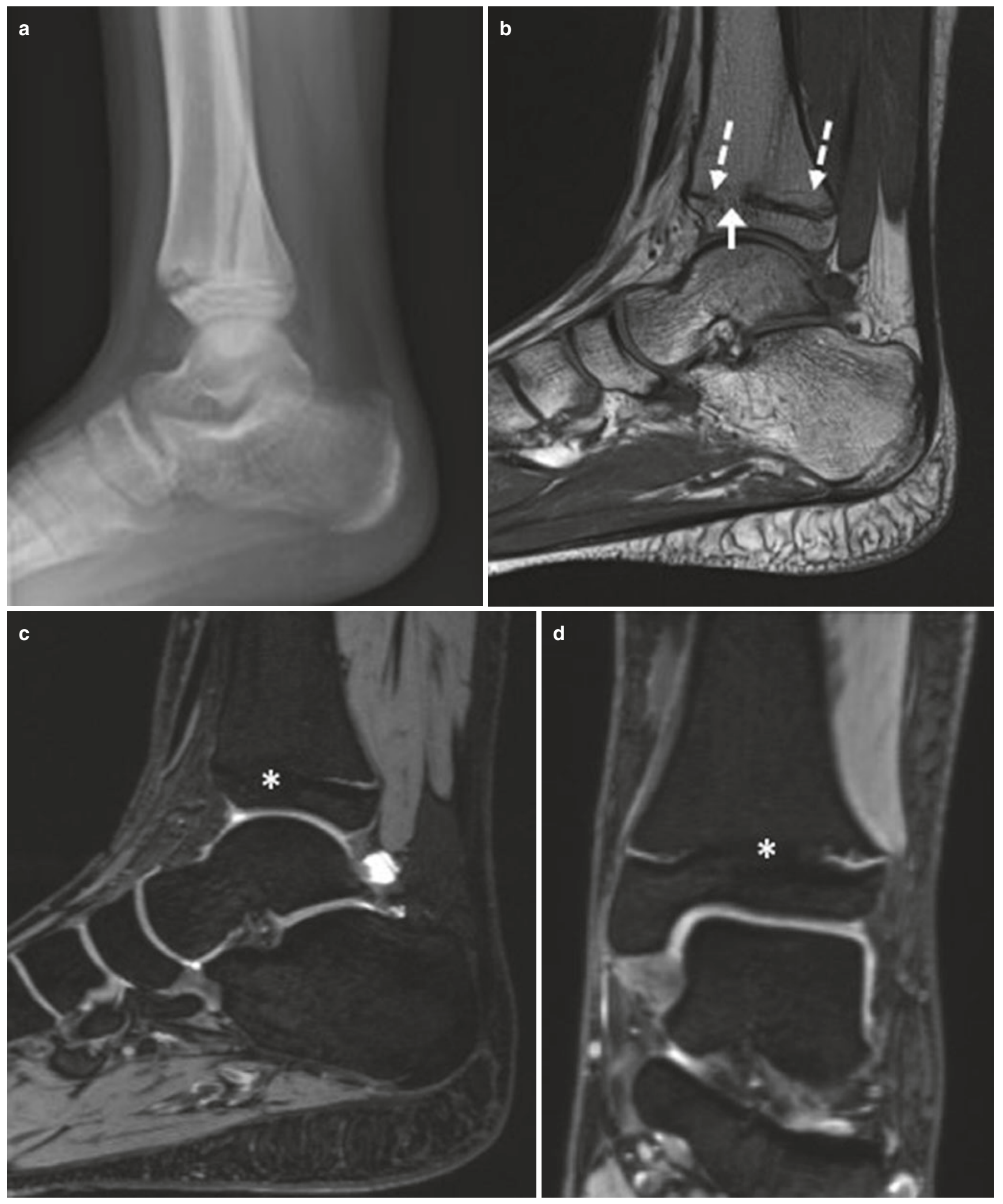

Fig. 19.7 (a-d) Salter-Harris II fracture with premature growth arrest. This 11-year-old girl landed on her inverted ankle when doing gymnastics on a trampoline. Lateral radiograph (a) at the time of injury shows the fracture plane through the physis anteriorly and extending through the metaphysis posteriorly. MR sagittal proton density (b) and 3D gradient echo steady state with fat suppression sagittal (c) and coronal (d) reformats 11 months after the fracture reveal a bone bridge with fatty marrow signal intensity (arrow, b) traversing the physis anteriorly, interrupting the normally high signal intensity physeal cartilage $(* \mathbf{c}, \mathbf{d})$. The growth recovering line (dashed arrow, b) tethered to the bone bridge indicates absent growth anteriorly and normal growth posteriorly 

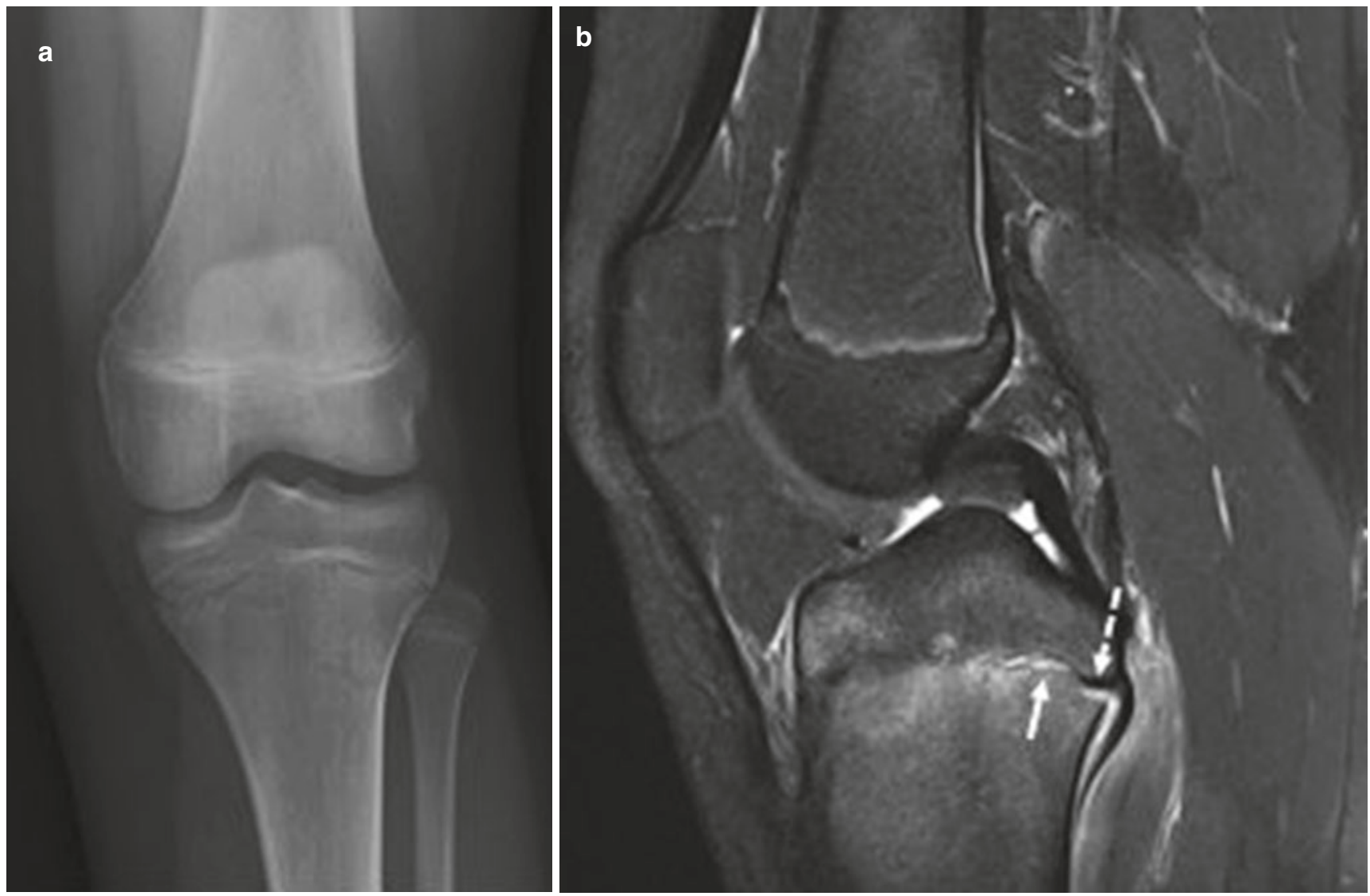

Fig. 19.8 (a, b) Radiographically occult proximal tibial fracture. AP radiograph (a) in a 13-year-old girl with lateral knee pain after colliding with another player in a soccer game shows no abnormality. MR sagittal T2-weighted image with fat suppression (b) obtained 1 week later for evaluation of persistent pain shows hyperintense fluid signal in the widened posterior physis (arrow) and marrow edema in the periphyseal epiphysis and metaphysis. Importantly, a low signal intensity linear structure within the posterior physis (dashed arrow) represents stripped periosteum which has become entrapped in the physis and may be an impediment to closed reduction extending into the distal femoral, proximal tibial, and proximal fibular metaphyses (Fig. 19.9). The abnormalities are medial or lateral depending upon the sport. The clinical symptoms and imaging findings of these chronic physeal stress injuries tend to resolve in 1 to 2 months with strict rest and/or immobilization. Continuation of athletic activity leads to persistent pain and growth disturbance with malalignment. Importantly, these abnormalities should not be confused with focal periphyseal edema (FOPE) zones commonly seen in the proximal tibia and distal femur in adolescence thought to be a manifestation of early physiologic physeal closure [18]. FOPE zones are predictably seen just prior to the normal physeal fusion, age range 11-15 years (Fig. 19.10). While FOPE zones may be symptomatic, they do not require treatment, and they resolve as normal physeal fusion progresses.

\section{Key Points}

\section{Physeal Injuries}

- Acute physeal fractures may lead to growth arrest with bony bridging across the open physis.

- Post-traumatic growth arrest is more dependent upon the specific anatomic location (distal femur, distal tibia, proximal tibia most common) than on the Salter-Harris type.

- Overuse or repetitive stress injury of the physis results in bands or tongues of unossified physeal cartilage extending into the metaphysis.

- Do not confuse normal physiologic FOPE zones with physeal injury. 

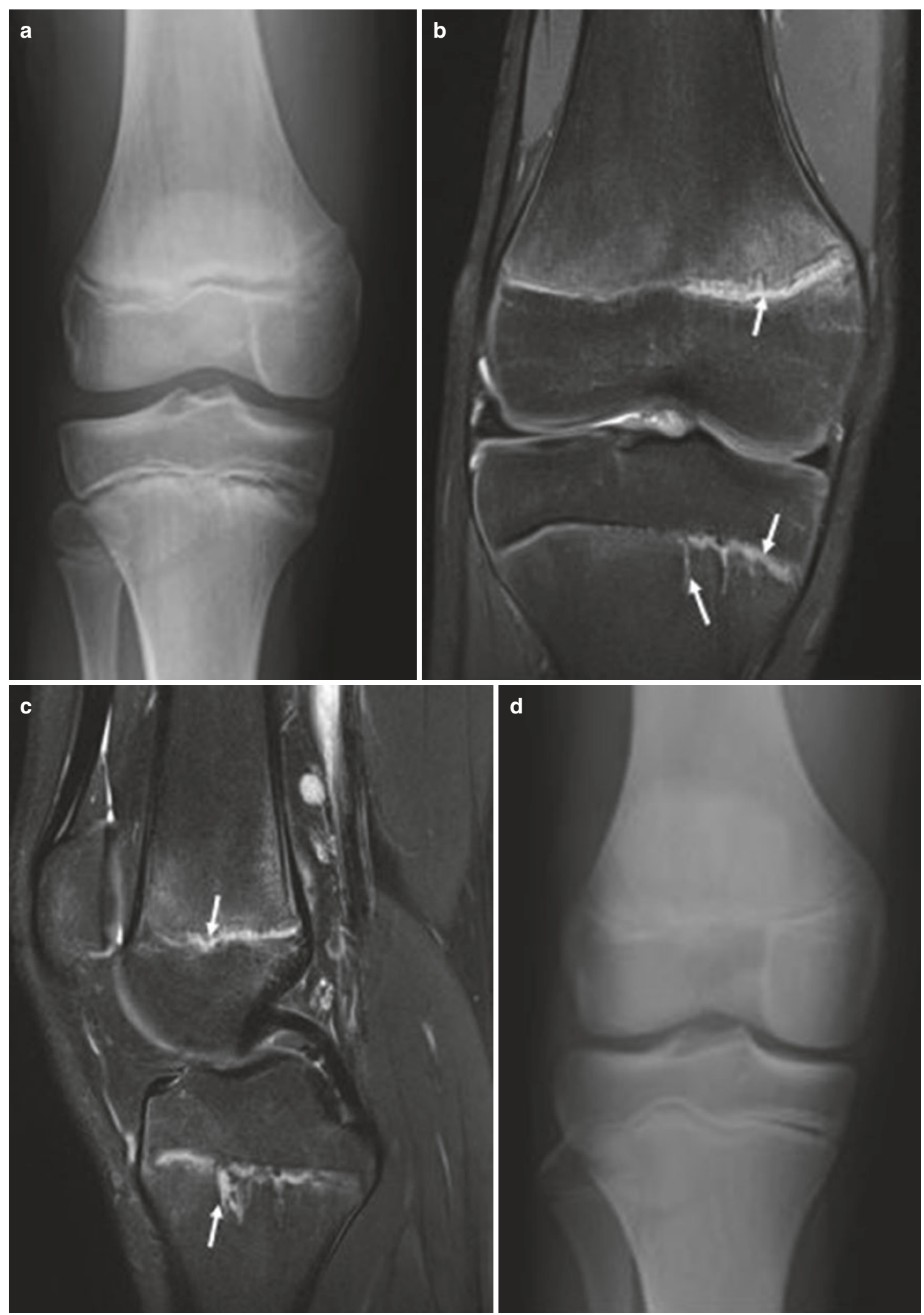

Fig. 19.9 (a-d) Overuse physeal stress injury. A 14-year-old ice hockey player with 3 months of knee pain. AP radiograph (a) shows medial physeal widening in the distal femur and proximal tibia. Coronal PD fat-suppressed (b) and sagittal T2-weighted fat-suppressed (c) images show medial physeal widening and hyperintensity (arrows).
The high signal reflects unossified physeal cartilage that seems to extend into the metaphysis. The cartilage may be band-like, as in the femur, or more spiculated and tongue-like, as in the tibia. The AP radiograph (d) obtained 3 months later after strict rest shows normalization of the physes 

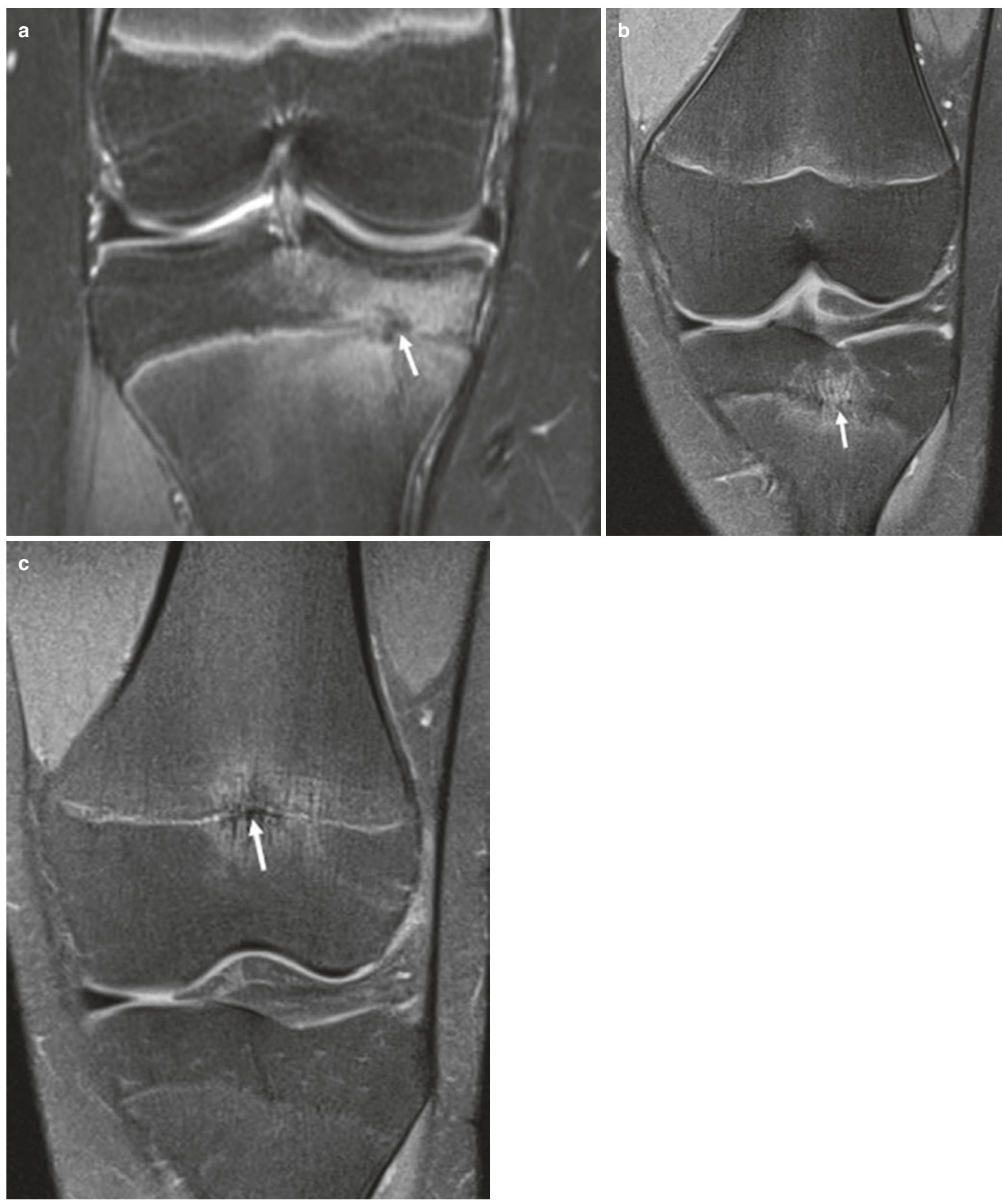

Fig. 19.10 (a-c) Focal periphyseal edema (FOPE) zones. Coronal PD fat-suppressed MR image (a) in a 13-year-old ballet dancer with medial knee pain shows marked bone marrow edema within the medial epiphysis and metaphysis centered around the fusing proximal tibial physis (arrow). Cor PD fat-suppressed MR image (b) in a 14-year-old female asymptomatic research volunteer shows similar but less intense edema around the fusing proximal tibial physis (arrow). One year later (c) the

tibial physis has fused normally and the edema has resolved. A new FOPE zone is present in the distal femoral physis centrally (arrow). This is consistent with the normal growth pattern-the proximal tibial physis fuses approximately 1 year prior to distal femoral fusion. Periphyseal edema should only be ascribed to this phenomenon in adolescents with normally fusing physes 


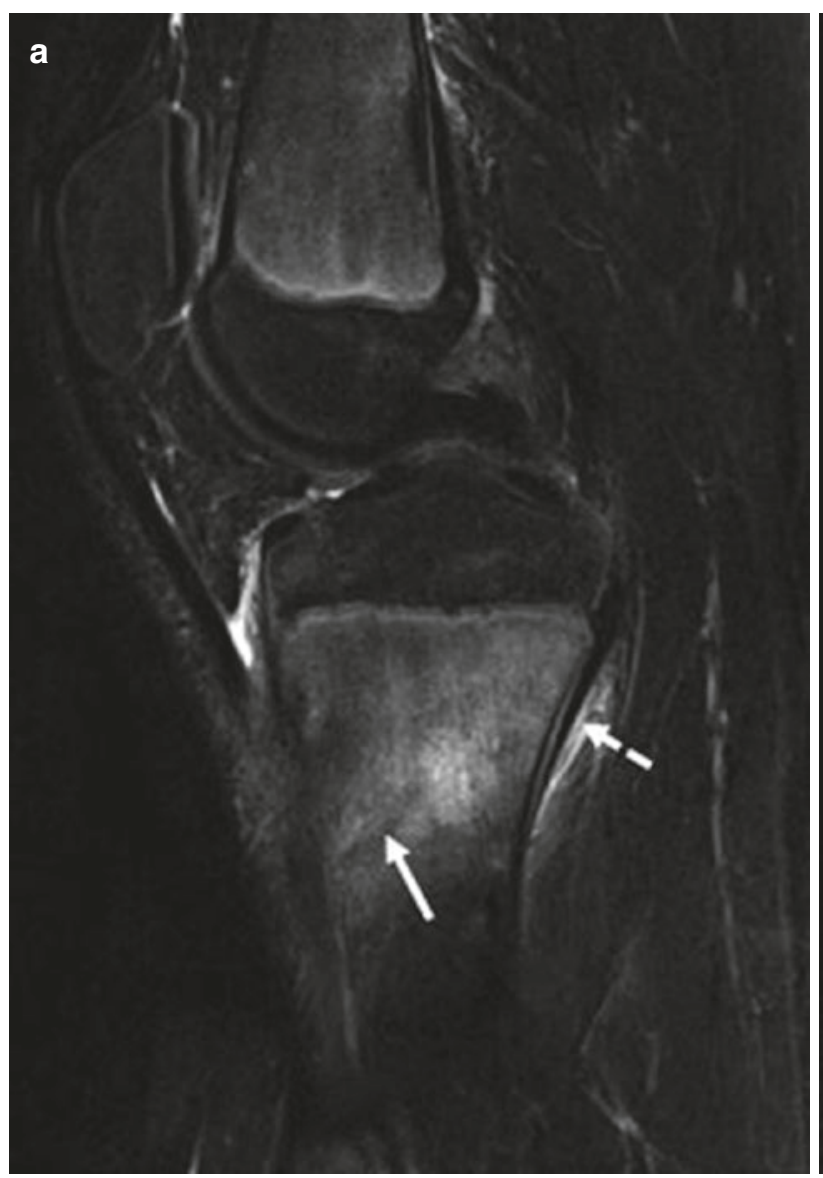

Fig. 19.11 (a, b) Tibial stress fracture. Sagittal T2 fat-suppressed MR image (a) in a 14-year-old boy with proximal tibial pain in the setting of increased lacrosse training shows metaphyseal marrow edema with a subtle low signal intensity oblique line centrally (arrow) and posterior

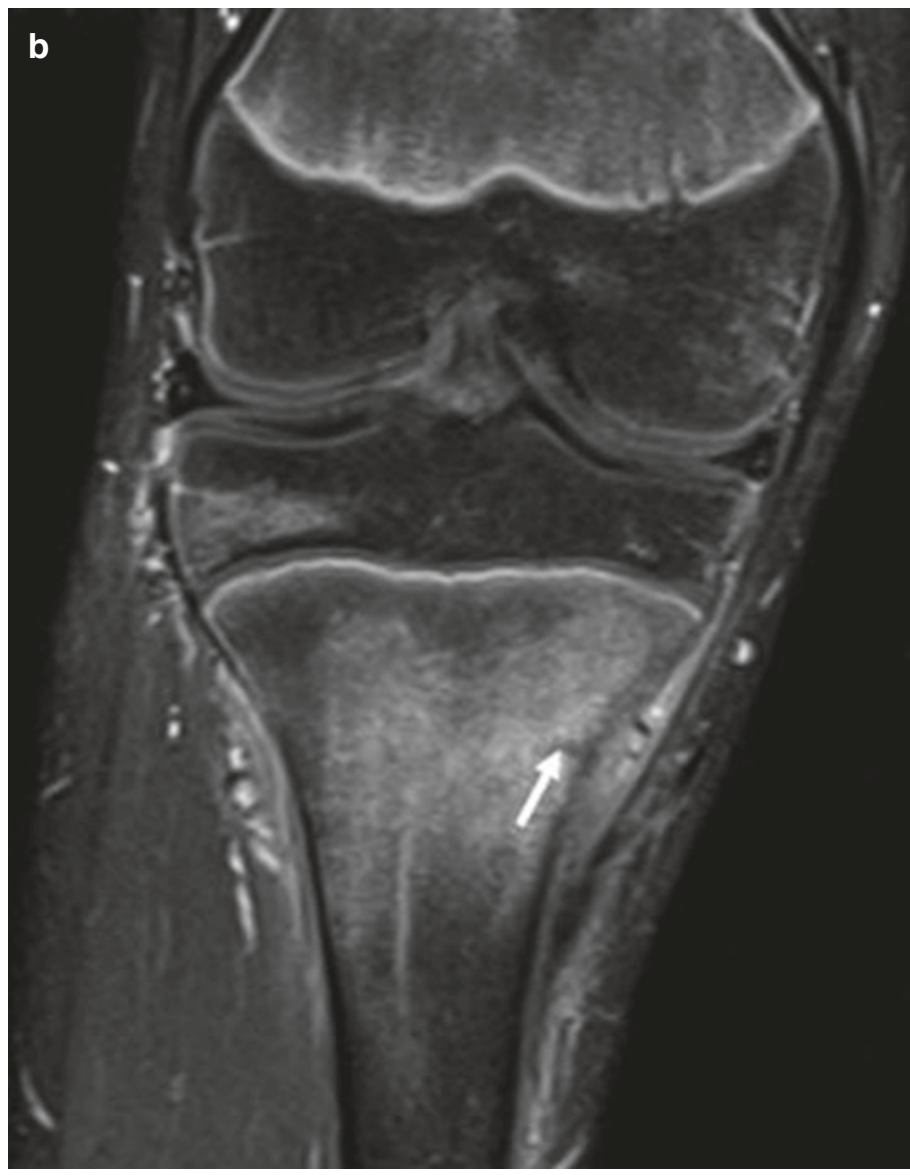

periosteal fluid (dashed arrow). The coronal T1 fat-suppressed postcontrast MR image (b) more clearly identifies the medial cortical fracture line (arrow). Contrast is generally unnecessary for stress injury evaluation but, when administered, nicely outlines fracture lines

\subsection{Long Bone Stress Injuries}

Stress fractures and stress reactions in children, once considered rare, are seen with increasing frequency presumably related to increased participation in athletics. Stress is the force applied to a bone that arises from weight-bearing and from muscular contractions. Lower muscle mass, decreased bone mineral density, and thinner cortices in addition to hormonal influences predispose the immature skeleton to stress injury. A 7-year prospective study of 6831 adolescent girls found a nearly $4 \%$ incidence of stress fractures with highimpact activities (running, basketball, cheerleading, and gymnastics) as significant risk factors [19]. Almost 50\% of all stress fractures in athletic children are tibial, with the remainder occurring in the fibula, femur, metatarsals, and tarsal bones. Unfortunately, the sensitivity of early radiographs is as low as $15 \%$ and delayed radiographs only reveal findings in $50 \%$ of patients. At MR imaging, STIR sequences and $\mathrm{T} 2$-weighted sequences with frequency selective fat saturation are exquisitely sensitive to the high signal intensity periosteal and marrow edema associated with stress reactions. A low signal intensity line within the high signal marrow edema allows the lesion to be upgraded to a stress fracture (Fig. 19.11). This line allows the lesion to be confidently identified as a stress fracture. Multidector CT with long-axis reformations can also be very helpful in delineating the occult fracture lucency.

\subsection{Concluding Remarks}

With the increased participation of children in athletics, radiologists are more frequently asked to assist in the diagnosis and management of acute and chronic injury to the growing skeleton. Initial radiographic assessment is often sufficient. However, we are increasingly expected to accurately evaluate soft tissue, ligamentous, tendinous, and cartilaginous injuries in competitive child athletes. MRI is often required for evaluation of injury to these radiolucent structures. Familiarity with the normal MR appearance of the growing 
musculoskeleton is essential to accurate interpretation of these imaging studies in young athletes. Accurate diagnosis and management are critical to mitigate the impact upon the growth mechanism and associated lifelong disability.

\section{Take Home Messages}

- MR depiction of normal musculoskeletal development must be differentiated from physeal and osteochondral injury.

- Overuse injuries in children are more common than previously realized.

- Growth disturbance associated with acute and overuse injuries can lead to lifelong morbidity.

\section{References}

1. König F. Ueber freie Körper in den Gelenken. Deutsche Zeitschrift für Chirurgie. 1888;27(1):90-109. https://doi.org/10.1007/ BF02792135.

2. Edmonds EW, Polousky J. A review of knowledge in osteochondritis dissecans: 123 years of minimal evolution from Konig to the ROCK study group. Clin Orthop Relat Res. 2013;471(4):1118-26. https://doi.org/10.1007/s11999-012-2290-y.

3. Ellermann J, Johnson CP, Wang L, Macalena JA, Nelson BJ, LaPrade RF. Insights into the epiphyseal cartilage origin and subsequent osseous manifestation of juvenile osteochondritis dissecans with a modified clinical MR imaging protocol: a pilot study. Radiology. 2017;282(3):798-806. https://doi.org/10.1148/ radiol.2016160071.

4. De Smet AA, Ilahi OA, Graf BK. Reassessment of the MR criteria for stability of osteochondritis dissecans in the knee and ankle. Skelet Radiol. 1996;25(2):159-63. https://doi.org/10.1007/ s002560050054.

5. Gebarski K, Hernandez RJ. Stage-I osteochondritis dissecans versus normal variants of ossification in the knee in children. Pediatr Radiol. 2005;35(9):880-6. https://doi.org/10.1007/ s00247-005-1507-6.

6. Laor T, Zbojniewicz AM, Eismann EA, Wall EJ. Juvenile osteochondritis dissecans: is it a growth disturbance of the secondary physis of the epiphysis? AJR Am J Roentgenol. 2012;199(5):11218. https://doi.org/10.2214/AJR.11.8085.

7. Laor T, Jaramillo D. MR imaging insights into skeletal maturation: what is normal? Radiology. 2009;250(1):28-38. https://doi. org/10.1148/radiol.2501071322.

8. Jaimes C, Chauvin NA, Delgado J, Jaramillo D. MR imaging of normal epiphyseal development and common epiphyseal disorders. Radiographics. 2014;34(2):449-71. https://doi.org/10.1148/ rg.342135070.

9. Oeppen RS, Connolly SA, Bencardino JT, Jaramillo D. Acute injury of the articular cartilage and subchondral bone: a common but unrecognized lesion in the immature knee. AJR Am J Roentgenol. 2004;182(1):111-7. https://doi.org/10.2214/ajr.182.1.1820111.

10. Varich LJ, Laor T, Jaramillo D. Normal maturation of the distal femoral epiphyseal cartilage: age-related changes at MR imaging. Radiology. 2000;214(3):705-9. https://doi.org/10.1148/radiology.2 14.3.r00mr20705.

11. Bedoya MA, Jaramillo D, Chauvin NA. Overuse injuries in children. Top Magn Reson Imaging. 2015;24(2):67-81. https://doi. org/10.1097/RMR.0000000000000048.

12. Merrow AC, Reiter MP, Zbojniewicz AM, Laor T. Avulsion fractures of the pediatric knee. Pediatr Radiol. 2014;44(11):1436-45; quiz 3-6. https://doi.org/10.1007/s00247-014-3126-6.

13. Meyers MH, Mc KF. Fracture of the intercondylar eminence of the tibia. J Bone Joint Surg Am. 1959;41-A(2):209-20; discussion $20-2$.

14. Salter RB, Harris WR. Injuries involving the epiphyseal plate. JBJS. 1963;45(3):587-622.

15. PetersonHA.Physealfractures:Part3.Classification.JPediatrOrthop. 1994;14(4):439-48. https://doi.org/10.1097/01241398-19940700000004

16. Chen J, Abel MF, Fox MG. Imaging appearance of entrapped periosteum within a distal femoral Salter-Harris II fracture. Skelet Radiol. 2015;44(10):1547-51. https://doi.org/10.1007/s00256-015-2201-x.

17. Laor T, Wall EJ, Vu LP. Physeal widening in the knee due to stress injury in child athletes. AJR Am J Roentgenol. 2006;186(5):1260 4. https://doi.org/10.2214/AJR.04.1606.

18. Zbojniewicz AM, Laor T. Focal Periphyseal Edema (FOPE) zone on MRI of the adolescent knee: a potentially painful manifestation of physiologic physeal fusion? AJR Am J Roentgenol. 2011;197(4):998-1004. https://doi.org/10.2214/AJR.10.6243.

19. Field AE, Gordon CM, Pierce LM, Ramappa A, Kocher MS. Prospective study of physical activity and risk of developing a stress fracture among preadolescent and adolescent girls. Arch Pediatr Adolesc Med. 2011;165(8):723-8. https://doi.org/10.1001/ archpediatrics.2011.34.

Open Access This chapter is licensed under the terms of the Creative Commons Attribution 4.0 International License (http://creativecommons. org/licenses/by/4.0/), which permits use, sharing, adaptation, distribution and reproduction in any medium or format, as long as you give appropriate credit to the original author(s) and the source, provide a link to the Creative Commons license and indicate if changes were made.

The images or other third party material in this chapter are included in the chapter's Creative Commons license, unless indicated otherwise in a credit line to the material. If material is not included in the chapter's Creative Commons license and your intended use is not permitted by statutory regulation or exceeds the permitted use, you will need to obtain permission directly from the copyright holder. 